Article

\title{
Numerical Validation of a Vortex Model against Experimental Data on a Straight-Bladed Vertical Axis Wind Turbine
}

\author{
Eduard Dyachuk * and Anders Goude \\ Division of Electricity, Department of Engineering Sciences, Uppsala University, Box 534, \\ Uppsala 751 21, Sweden; E-Mail: anders.goude@angstrom.uu.se \\ * Author to whom correspondence should be addressed; E-Mail: eduard.dyachuk@angstrom.uu.se; \\ Tel.: +46-18-471-5849.
}

Academic Editor: Frede Blaabjerg

Received: 3 August 2015 / Accepted: 13 October 2015 / Published: 20 October 2015

\begin{abstract}
Cyclic blade motion during operation of vertical axis wind turbines (VAWTs) imposes challenges on the simulations models of the aerodynamics of VAWTs. A two-dimensional vortex model is validated against the new experimental data on a $12-\mathrm{kW}$ straight-bladed VAWT, which is operated at an open site. The results on the normal force on one blade are analyzed. The model is assessed against the measured data in the wide range of tip speed ratios: from 1.8 to 4.6. The predicted results within one revolution have a similar shape and magnitude as the measured data, though the model does not reproduce every detail of the experimental data. The present model can be used when dimensioning the turbine for maximum loads.
\end{abstract}

Keywords: wind turbine; vertical axis turbine; force; measurement; open site; simulation; vortex model; dynamic stall

\section{Introduction}

The interest in vertical axis wind turbines (VAWTs) as an alternative to the conventional horizontal axis wind turbines (HAWTs) has been growing in recent years [1]. This is due to the potential of VAWTs to decrease the cost of wind energy [2,3]. VAWTs have several advantages over HAWTs: the generator of a VAWT can be located at the ground level, therefore excluding the concerns over the mass and size of the generator. The advantage of the lower center of mass (compared to HAWTs) is very important for floating platforms [4]. Additionally, the yawing mechanism is excluded for VAWTs, since they are 
omni-directional. Thus, the simplicity of the concept with only a few moving parts is one of the main advantages of VAWTs over HAWTs.

The complex aerodynamics of VAWTs imposes significant challenges on the simulation models. The flow velocity at the blades of the VAWT changes constantly during the turbine rotation, which causes the angle of attack to change during every revolution. The magnitude of the variation of the angle of attack increases with the decreased turbine tip speed ratio (TSR). At low TSRs, the blades of the VAWT experience the event of dynamic stall, which is associated with the rapid decrease in the lift and the increase in the drag force, reducing the torque on the turbine. At high TSRs, the flow velocity when passing through the turbine is decreased more than at low TSRs, and therefore, the flow expansion is prevailing at high TSRs.

The simulation models for VAWTs can be divided into three groups. The first group includes the finite element method (FEM) or the finite volume method (FVM), which are used to solve the Navier-Stokes equations within the commonly-available software for computational fluid dynamics (CFD). The second method is based on the vorticity equation, and the models are usually referred to as vortex models. The third method is based on the momentum conservation principle, and one of the most common and advanced momentum models is the double multiple streamtube model. The overview of the aerodynamic models for VAWTs can be found in [5-7]. A two-dimensional (2D) vortex model combined with the Leishman-Beddoes-type dynamic stall model is used in this study. The model combines the vorticity equation with experimental data, which results in the high computational speed of the model. This model gives the flow velocity field and is time dependent.

There is a lack of experimental data on the blade forces during one revolution for VAWTs. A series of the experiments were conducted by the Sandia National Laboratories in the 1980s, where VAWTs with curved blades (Darrieus turbines) were operated at open sites [8-10]. Other measured data concern small vertical axis turbines operating in wind tunnels or towing tanks with low operational Reynolds numbers [11,12]. Since the force coefficients are dependent on the Reynolds number, the aerodynamics of large turbines are different from the aerodynamics of turbines operated in wind tunnels or towing tanks. Thus, due to high Reynolds numbers, the measured data from the Sandia National Laboratories are still used for the validation of simulation models [13-16].

This study assesses measurements from 2014 on a straight-bladed VAWT, which operates at an open site with the average Reynolds number of 300,000 [17,18]. A study on the power coefficient $\left(C_{P}\right)$ of this VAWT from 2011 has shown that the turbine reaches its maximum $C_{P}$ of 0.29 at the TSR of 3.3 [19]. However, the turbine diameter has increased from 6 to $6.5 \mathrm{~m}$ after mounting the load cell assembly, and the power coefficient is expected to be slightly different for the modified turbine as the turbine solidity has decreased. New experimental data on the normal forces on this VAWT are presented. The goal of the study is to describe the simulation model and to validate it against the experimental data. The normal forces are compared for the range of TSRs from 1.8 to 4.6, covering the dynamic stall region and the region of high flow expansion. The results and the capability of the model are analyzed. 


\section{Experimental Data}

The measurement data used in this study are obtained from the 12-kW VAWT located outside Uppsala, Sweden. The experimental method and the obtained force data are described in detail in $[17,18]$. It follows that the measured normal force was periodic and consistent, while the tangential force response was highly disturbed by the turbine dynamics. Hence, only the normal force measurements can be considered suitable for usage in this validation. The studied VAWT is a 3-bladed H-rotor turbine with a radius of $3.24 \mathrm{~m}$ and a blade length of $5 \mathrm{~m}$; Figure 1 . The blades are pitched outwards 2 degand have the NACA0021profile with a chord length of $0.25 \mathrm{~m}$ at the middle of the blade. The turbine with assembled force sensors is shown in Figures 2 and 3. The sensors are single-axis load cells, which measure tension and compression at a point load. The rotational speed of this turbine can be kept at a constant level [17,19], and the normal force is estimated using the notations from Figures 2 and 3 as the following:

$$
F_{N}=F_{0}+F_{1}+F_{2}+F_{3}-F_{C}
$$

where $F_{C}$ is the centrifugal force:

$$
F_{C}=m \Omega^{2} L_{C}
$$

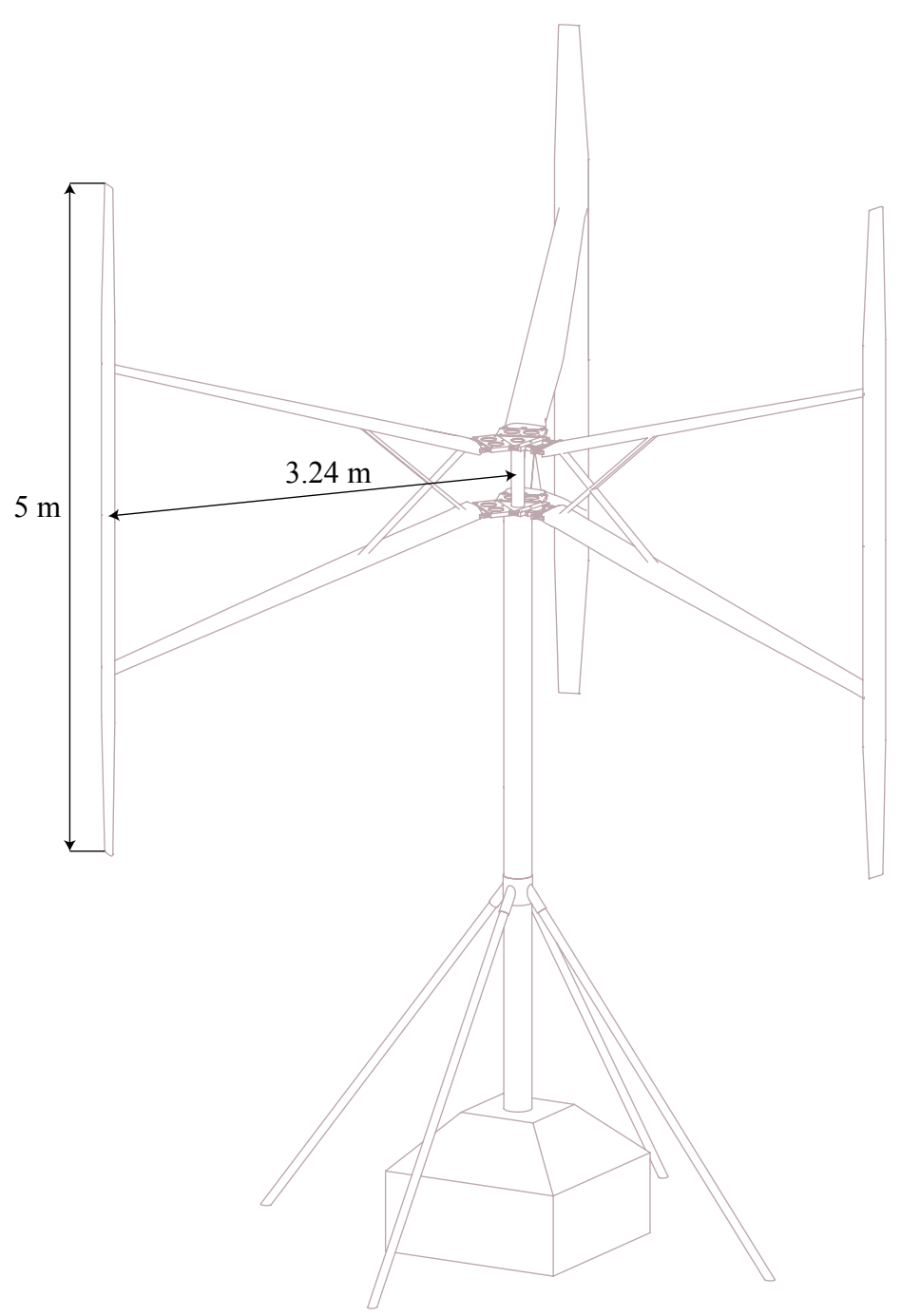

Figure 1. The vertical axis wind turbine (VAWT) used for the experiment. 


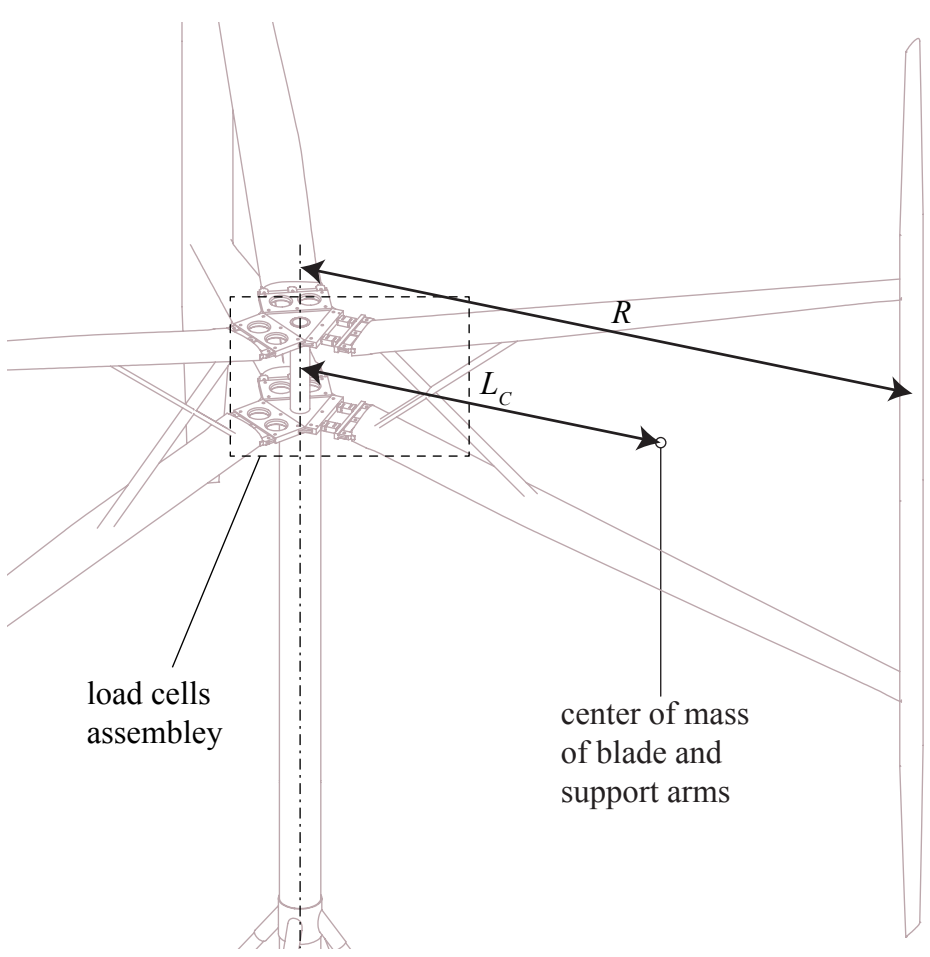

Figure 2. Load cells installed on the VAWT.

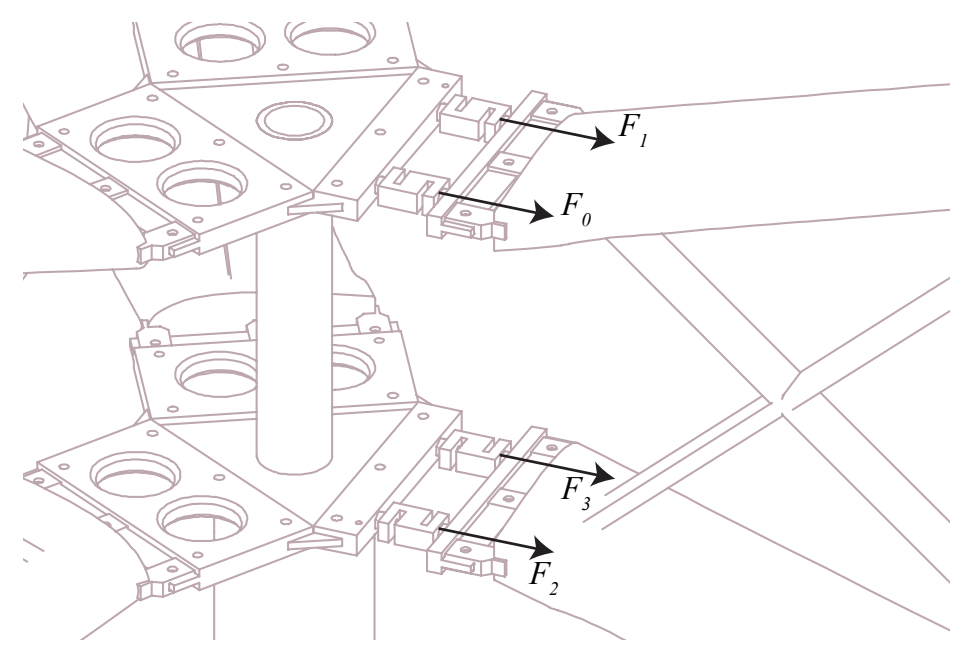

Figure 3. The assembly of the load cells. The notation of the measured forces.

Here, $m=35.79 \mathrm{~kg}$ is the mass of the blade and support arms, $\Omega$ is the turbine rotational speed and $L_{C}=1.83 \mathrm{~m}$ is the distance from the axis of rotation to the center of mass of the blade assembled with the support arms. $F_{0}, F_{1}, F_{2}$ and $F_{3}$ are the measured forces.

Due to varying weather conditions, the force measurements were analyzed only for times with steady wind flow conditions. The relative standard deviation (RSD) was used to quantify wind flow variations:

$$
R S D(x)=\left(\frac{1}{n} \sum_{j=1}^{n}\left(x_{j}-\langle x\rangle\right)^{2}\right)^{\frac{1}{2}} \frac{1}{\langle x\rangle} \times 100 \%
$$

where $\langle x\rangle$ denotes the average value of variable $x$. 
The measured data were divided into 24 s-long bins: $16 \mathrm{~s}$ to stabilize the turbine wake ("wake time", corresponding to 10 revolutions at $40 \mathrm{rpm}$ ) followed by $8 \mathrm{~s}$ of steady flow operation ("disk time", 5 revolutions at $40 \mathrm{rpm}$ ). Wind flow was considered as steady for bins with the RSD of the asymptotic wind velocity $V_{\infty}$ of $R S D_{\text {wake }}\left(V_{\infty}\right) \leq 10 \%, R S D_{\text {disk }}\left(V_{\infty}\right) \leq 5 \%$ and the RSD of wind direction $V$ dir of $R S D_{\text {wake,disk }}(V d i r) \leq 1 \%$. This definition of the steady wind flow conditions is documented in [18]. Variations of the wind speed during the steady conditions are illustrated in Figure 4.

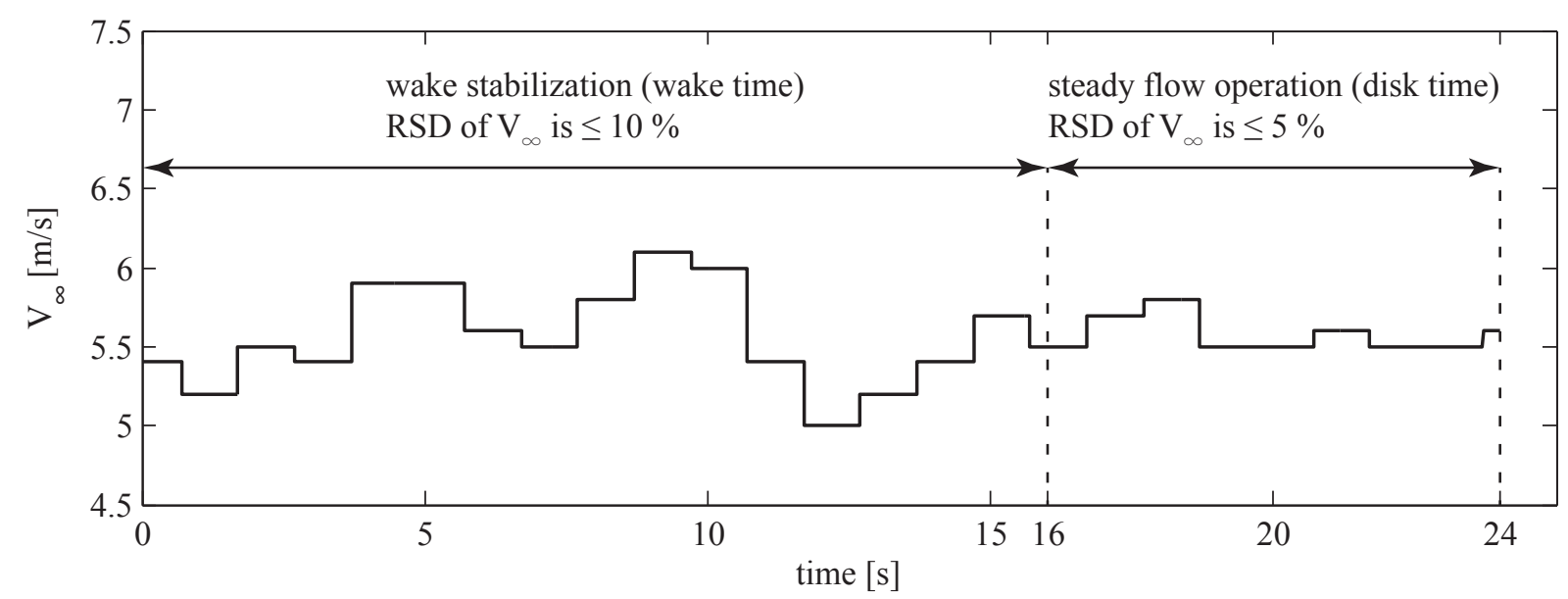

Figure 4. Allowed variations of the asymptotic wind velocity inside a bin with the steady wind flow conditions.

The normal force during one revolution is obtained as the average response over 5 revolutions with steady wind flow. The operational TSR is estimated as:

$$
\lambda=\frac{\langle\Omega\rangle R}{\left\langle V_{\infty}\right\rangle}
$$

where $\Omega$ is the turbine rotational speed. The average values of $\Omega$ and $V_{\infty}$ are taken taken over time with steady wind flow.

The analysis of the measurement accuracy has shown that the maximum error of the measured normal force is a function of the turbine rotational speed:

$$
\Delta F_{N}= \pm\left(0.0049 \Omega_{\mathrm{rpm}}^{2}+0.072 \Omega_{\mathrm{rpm}}+23\right)
$$

where $\Omega_{\mathrm{rpm}}$ is the rotational speed in rpm. For the details regarding the measurement accuracy, the reader is referred to $[17,18]$. The air density $\rho$ is calculated for the measured air temperature, pressure and humidity according to [17]. The kinematic viscosity $\nu$ is estimated as the function of the measured air temperature [20]. 


\section{Simulation Model}

This section presents the vortex method together with the dynamic stall model to predict the blade forces of the VAWT. The sign notation of the forces together with the blade azimuth angle are defined in Figure 5.

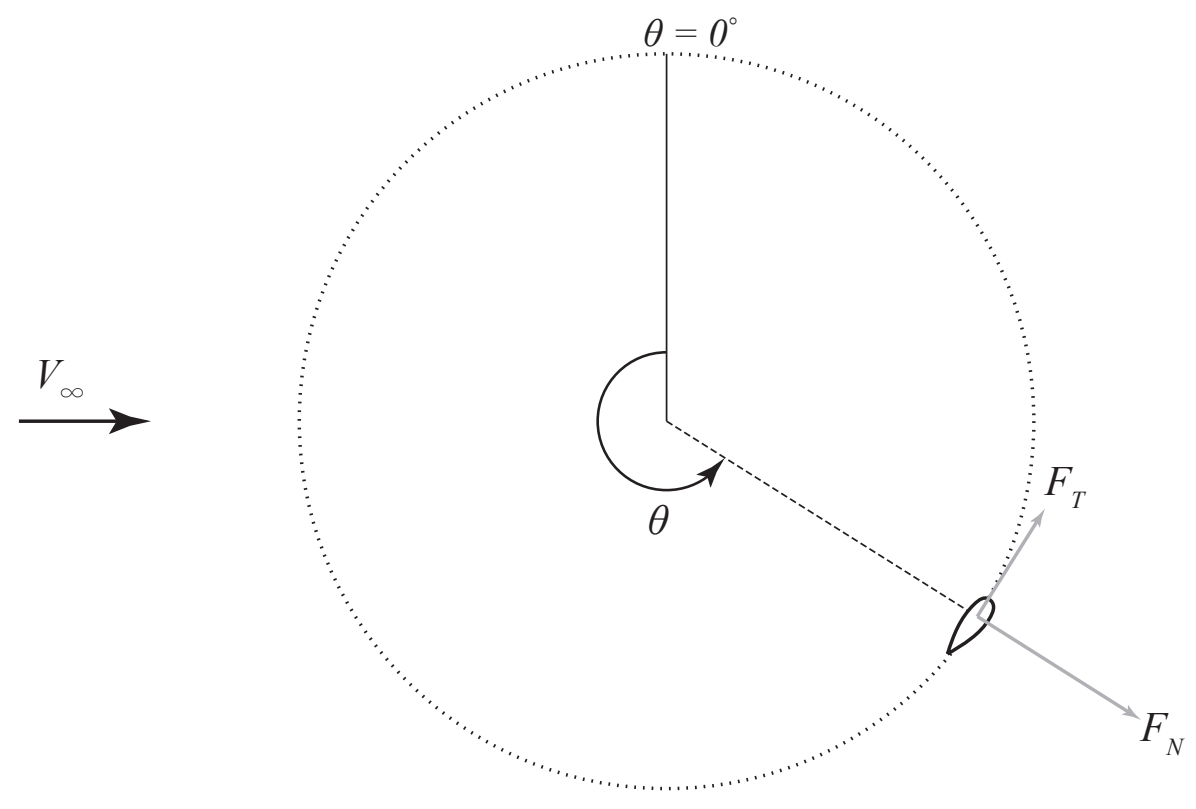

Figure 5. The sign convention of the normal and tangential forces. The counter-clockwise direction of the blade azimuth angle $\theta$ is defined as positive.

\subsection{Vortex Model of the Turbine}

The vortex method is commonly used for solving the flow of vertical axis turbines. The main idea behind the model is to use the vorticity as the discretization variable, instead of the velocity. The vorticity is obtained by taking the curl of the flow velocity:

$$
\vec{\omega}=\nabla \times \vec{V}
$$

and similarly, the vorticity equation is obtained from the curl of the Navier-Stokes equations:

$$
\frac{\partial \vec{\omega}}{\partial t}+(\vec{V} \cdot \nabla) \vec{\omega}=(\vec{\omega} \cdot \nabla) \vec{V}+\nu \nabla^{2} \vec{\omega}
$$

which in the two-dimensional case becomes:

$$
\frac{\partial \vec{\omega}}{\partial t}+(\vec{V} \cdot \nabla) \vec{\omega}=\nu \nabla^{2} \vec{\omega}
$$

The current implementation uses a free vortex model, where the individual vortices are used as discretization variables. The flow velocity is obtained from the model as a superposition of the potential flow solution and the contribution from the vortices:

$$
\vec{V}=\nabla \phi+\vec{V}_{\omega}
$$


Under the assumption that the turbine is not confined with walls and that that the blade is approximated as a single point, the potential flow solution $\nabla \phi$ is equal to the asymptotic flow velocity $V_{\infty}$. For the two-dimensional vortex method, according to the Biot-Savart law and using the complex numbers, the velocity contribution from vortices $\vec{V}_{\omega}$ at position $r$ becomes:

$$
V_{\omega}(r)=\sum_{k=1}^{N_{\nu}} \frac{i \Gamma_{k}}{2 \pi} \frac{1}{\left(\bar{r}-\bar{r}_{k}\right)}\left(1-e^{-\frac{\left|\bar{r}-\bar{r}_{k}\right|^{2}}{\varepsilon^{2}}}\right)
$$

Here, $r_{k}$ is the position and $\Gamma_{k}$ is the circulation of vortex $k\left(\bar{r}_{k}\right.$ denotes the complex conjugate of $\left.r_{k}\right)$; $N_{v}$ is the total number of released vortices. The Gaussian kernel $\left(1-e^{-\frac{\left|r-r_{k}\right|^{2}}{\varepsilon^{2}}}\right)$ is used to avoid the non-physical divergences when $r$ approaches $r_{k}$ [21].

The relative wind velocity at the turbine blades is a vector sum of the flow velocity at the blade due to its own motion, $-\vec{V}_{b}$ and the flow velocity $\vec{V}$ :

$$
\vec{V}_{r e l}=\vec{V}-\vec{V}_{b}
$$

where the velocity $\vec{V}$ is given by Equations (9) and (10), and the blade tangential velocity $\vec{V}_{b}$ is:

$$
\overrightarrow{V_{b}}=\Omega R \hat{t}
$$

Here, $\hat{t}$ is the unit vector in the tangential direction with the same sign convention as the blade azimuth angle $\theta$ in Figure 5. In the Lagrangian formulation, the vortices are allowed to drift with the flow velocity. Neglecting the viscosity outside the boundary layers of the blades, the vortices are propagated according to:

$$
\frac{d \vec{r}}{d t}=\vec{V}(\vec{r})
$$

where the velocity $\vec{V}$ is calculated with Equations (9) and (10). The velocities at each vortex position can efficiently be evaluated using the fast multipole method [22], and the current work uses the implementation described in [23].

Even though the vortex method can solve the entire flow, a full solution of the boundary layer will require a high computational effort. To significantly improve the speed, a dynamic stall model of the blade force coefficients is used to obtain the lift and drag coefficients; see Section 3.2. The dynamic stall model requires the flow velocity and the angle of attack to be calculated. The absolute value of the relative flow velocity $V_{\text {rel }}=\left|\vec{V}_{\text {rel }}\right|$ is calculated with Equations (9) to (11) and is used as an input to the blade force model. To properly handle the flow curvature effects from the rotating motion of the turbine, the blade geometry has to be modeled. In the current implementation, the blade is modeled with linear panels with the linear distribution of vorticity according to [5,24]. Using these panels, the bound circulation of the blades $\Gamma_{\text {blade }}$ can be determined by enforcing the no-penetration boundary condition on the surface of airfoil and the Kutta condition on the trailing edge. This circulation can be used to calculate the corresponding angle of attack:

$$
\alpha=p\left(\arcsin \left(\frac{\Gamma_{\text {blade }}}{\pi c V_{\text {rel }}}\right)-\alpha_{0}\right)
$$

where $c$ is the blade chord length and parameters $p$ and $\alpha_{0}$ are determined by matching the bound circulation $\Gamma_{\text {blade }}$ with the known angle of attack from the steady-state potential flow solutions. 
The procedure of calculating the angle of attack is described in greater detail in [25], and the method denoted "explicit method" is the one implemented here. This method adds the released vortex into the panel equations and solves for the Kutta condition together with the conservation of circulation, i.e., the total circulation of the released vortex, and the bound circulation should equal the bound circulation from the previous time step.

With the velocity $V_{\text {rel }}$, the angle of attack $\alpha$ and its time derivative $\dot{\alpha}$, the lift and the drag force coefficients $\left(C_{L}\right.$ and $\left.C_{D}\right)$ are obtained within the blade force model for an airfoil profile with a given chord length $c$ and the Reynolds number (Section 3.2, Equation (31)). Please note that only symmetrical four-digit NACA airfoils are implemented in this work. The kinematic viscosity, obtained from the weather data (Section 2), is used in the model to estimate the local Reynolds number. The lift force coefficient from the blade force model can then be used to calculate the effective bound circulation $\Gamma_{d s}$ with the Kutta-Joukowski lift formula:

$$
\Gamma_{d s}=\frac{1}{2} C_{L} c V_{r e l}
$$

Due to conservation of circulation, a vortex has to be released from the trailing edge of the blade each time step $\Delta t$ with a strength $\Gamma_{\text {released }}$ corresponding to the change of circulation between the time steps:

$$
\Gamma_{\text {released }}=\Gamma_{d s, n-1}-\Gamma_{d s, n}
$$

where subscripts $n$ and $n-1$ stand for current and previous time steps. The position of the released vortex is chosen as $0.5 \Omega R \Delta t$ behind the trailing edge. In the case of dynamic stall, the bound circulation of the blade will be reduced, i.e., $\Gamma_{d s}<\Gamma_{\text {blade }}$. This means that the blade will no longer fulfil the Kutta condition, which then would give infinite flow velocities at the trailing edge. Therefore, during the evaluation of the vortex velocities, it is chosen to approximate the blade as a single point vortex (located at a quarter-chord position) that can be evaluated with Equation (10). This approximation also increases the computational speed (compared to modeling the blades with panels) as no panel to vortex interactions have to be calculated. This approximation is evaluated in [25], and the results show that the difference between approximating the blade with a point vortex or with panels is very small; hence, the approximation is reasonable.

All simulations were performed for 100 turbine revolutions to ensure convergence in the results. This value was chosen from the convergence studies performed in [26]. One hundred twenty time steps were performed for each revolution, and the turbine was kept at a constant rotational speed for the entire simulation. The normal force was calculated based on the obtained lift and drag coefficients:

$$
F_{N}=\frac{1}{2} \rho A_{\text {blade }} V_{r e l}^{2}\left(C_{L} \cos \varphi+C_{D} \sin \varphi\right)
$$

with blade area $A_{\text {blade }}$ and air density $\rho$. The angle $\varphi$ is the angle of the relative flow velocity vector $\vec{V}_{\text {rel }}$. The force values are presented from the last revolution of the simulations. An overview of the vortex model algorithm is illustrated in Figure 6, where the most important steps are highlighted. 


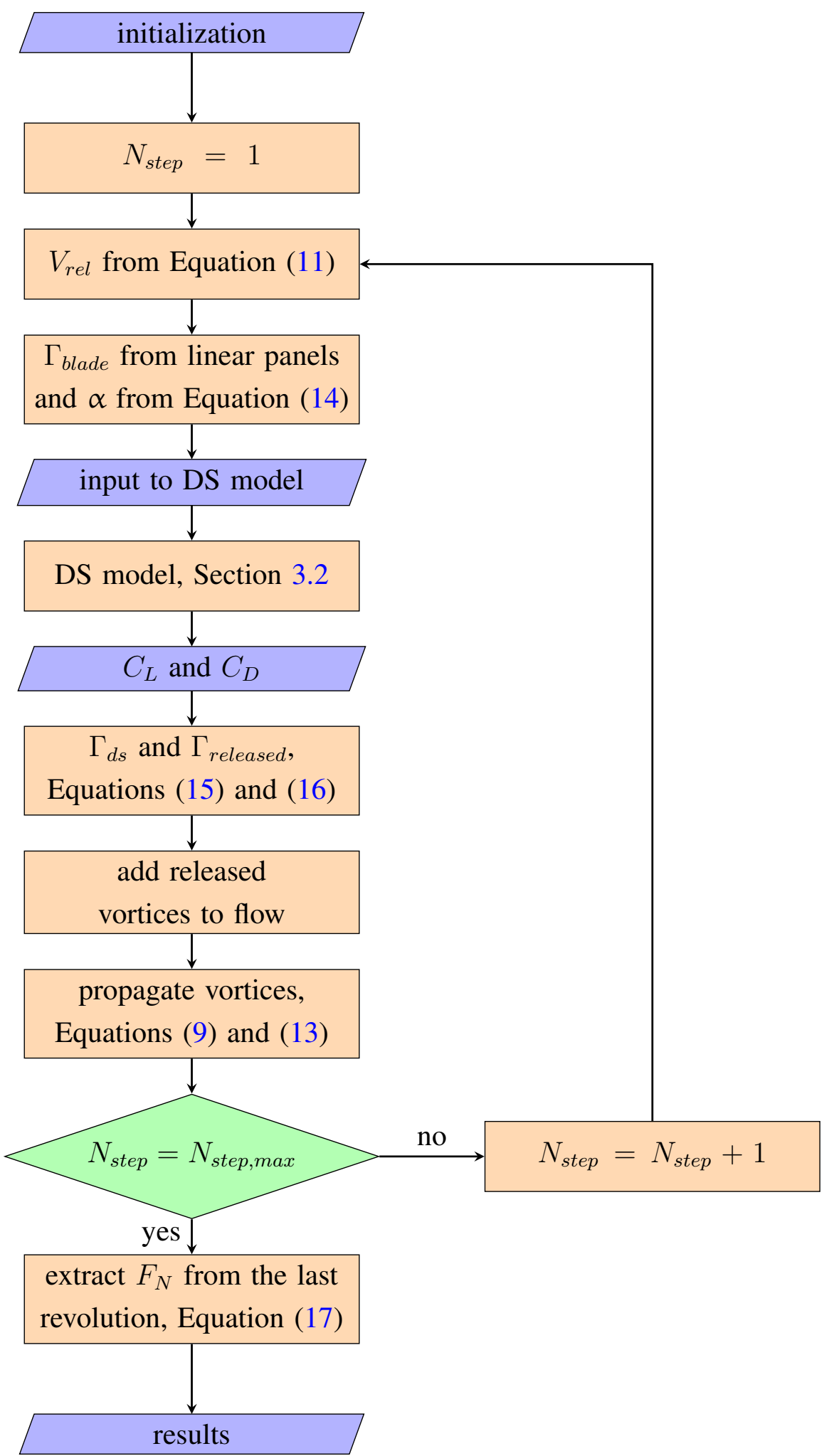

Figure 6. Flow chart of the vortex model combined with the dynamic stall (DS) model. $N_{\text {step }}$ is the current time step, and $N_{\text {step,max }}$ is the maximum number of time steps. $N_{\text {step, } \max }=12,000$, corresponding to 120 time steps per each of 100 revolutions. 


\subsection{Dynamic Stall Modeling}

The model originally developed by Leishman and Beddoes [27,28] with modifications for the conditions of VAWTs [29,30] is used for the modeling of the dynamic stall. The dynamic stall model uses experimental data of the lift and the drag coefficients for steady flow over airfoils [31]. This model was combined with the vortex model for a single pitching wing and showed reasonable agreement with experimental data on the pitching airfoils [25]. The inputs to the models are the angle of attack $\alpha$ and its rate of change $\dot{\alpha}$, the velocity $V_{\text {rel }}$, the chord length $c$, the Reynolds number and the blade airfoil. These inputs are obtained within the vortex model. The outputs of the dynamic stall model are the lift and the drag coefficients $\left(C_{L}\right.$ and $C_{D}$, respectively). The main principles of the model are described in this section, and the reader is referred to $[25,29]$ for the details, including the empirical parameters.

The Leishman-Beddoes dynamic stall model consists of three parts: unsteady attached flow, dynamic stall onset and unsteady separated flow part. The unsteady attached flow solution comprises impulsive and circulatory loading, which are caused by unsteady boundary vortex and the changes in the angles of attack. The change of the flow velocity due to the vortex contribution is already taken into account by the vortex model. Thus, the unsteady attached flow part of the Leishman-Beddoes model is not used when combined with the vortex model. This method is identical to the one described in [25].

A delay in the pressure response is represented by the further lag in the angle of attack:

$$
\alpha_{n}^{\prime}=\alpha_{n}-D_{\alpha_{n}}
$$

where $D_{\alpha}$ is the deficiency function:

$$
D_{\alpha_{n}}=D_{\alpha_{n-1}} \exp \left(-\frac{\Delta s}{T_{\alpha}}\right)+\left(\alpha_{n}-\alpha_{n-1}\right) \exp \left(-\frac{\Delta s}{2 T_{\alpha}}\right)
$$

Here, $T_{\alpha}$ is an empirically-derived constant, and its values for the symmetrical NACA-airfoils are found in [29]. For the NACA0021 airfoil, $T_{\alpha}=6.30$. The non-dimensional time step $\Delta s$ is calculated as:

$$
\Delta s=\frac{2 V_{r e l} \Delta t}{c}
$$

where $V_{\text {rel }}$ is the relative flow velocity obtained from Equation (11).

A critical angle of attack is defined to represent the onset of the dynamic stall:

$$
\alpha_{c r_{n}}= \begin{cases}\alpha_{d s 0} & \left|q_{n}\right| \geq q_{0} \\ \alpha_{s s}+\left(\alpha_{d s 0}-\alpha_{s s}\right) \frac{\left|q_{n}\right|}{q_{0}} & \left|q_{n}\right|<q_{0}\end{cases}
$$

with the reduced pitch rate $q$ as:

$$
q_{n}=\frac{\dot{\alpha_{n}} c}{2 V_{r e l}}
$$

Here, $q_{0}$ is the reduced pitch rate, which delimits the quasi-steady stall and the dynamic stall; $q_{0}=0.01 . \alpha_{d s 0}$ and $\alpha_{s s}$ are the critical static stall onset angle and the static stall angle, respectively, $\alpha_{d s 0}=17.91^{\circ}$ and $\alpha_{s s}=14.33^{\circ}$ for NACA0021 [29]. The dynamic stall condition is defined as when the delayed angle of attack $\alpha^{\prime}$ exceeds the critical angle of attack $\alpha_{c r}$ :

$$
\left|\alpha^{\prime}\right|>\alpha_{c r} \rightarrow \text { stall }
$$


The unsteady separated flow part includes the trailing edge and the leading edge vortex separation. The trailing edge separation is associated with the delay in the convection of the flow separation point over the surface of the airfoil. It is represented via Kirchhoff's flow approximation:

$$
f_{n}^{\prime}= \begin{cases}1-0.4 \exp \left(\frac{\left|\alpha_{n}^{\prime}\right|-\alpha_{1}}{S_{1}}\right) & \left|\alpha_{n}^{\prime}\right|<\alpha_{1} \\ 0.02+0.58 \exp \left(\frac{\alpha_{1}-\left|\alpha_{i}^{\prime}\right|}{S_{2}}\right) & \left|\alpha_{n}^{\prime}\right| \geq \alpha_{1}\end{cases}
$$

where $f^{\prime}$ is the delayed separation point and $S_{1}, S_{2}$ and $\alpha_{1}$ are the empirically-derived constants, which are based on the local Reynolds number and the airfoil profile and are found in [29]. In addition to the pressure response delay (which is represented by $\alpha^{\prime}$, Equation (18)), a further delay in the flow separation point is present in order to account for the time-dependent boundary layer:

$$
f_{i}^{\prime \prime}=f_{i}^{\prime}-D_{f_{i}}
$$

where $D_{f_{n}}$ is:

$$
D_{f_{n}}=D_{f_{n-1}} \exp \left(-\frac{\Delta s}{T_{f}}\right)+\left(f_{n}^{\prime}-f_{n-1}^{\prime}\right) \exp \left(-\frac{\Delta s}{2 T_{f}}\right)
$$

Here, $T_{f}=3$, which is the empirically-derived constant [29]. The normal force coefficient for the unsteady separated flow conditions before the dynamic stall onset is:

$$
C_{N_{n}}^{f}=C_{N_{\alpha}} \alpha_{n}\left(\frac{1+\sqrt{f_{n}^{\prime \prime}}}{2}\right)^{2}
$$

where $C_{N_{\alpha}}$ is the slope of the normal force coefficient at the static conditions, and it is based on the airfoil and the Reynolds number [29].

When the dynamic stall condition is met (Equation (23)), the leading edge vortex forms and propagates towards the trailing edge and then releases. This vortex convection is represented by an increase in the lift force (sometimes referred to as the vortex lift) during the vortex propagation and followed by a drop in the lift force when the vortex releases. The vortex lift is calculated as the follows:

$$
C_{N_{n}}^{v}=B_{1}\left(f_{n}^{\prime \prime}-f_{n}\right) V_{x}
$$

where $f$ is the static separation point and $B_{1}$ and $V_{x}$ are parameters, which are based on the local Reynolds number and the blade airfoil and are found in [29].

The total normal force coefficient is the sum of the unsteady normal force coefficient and the vortex lift:

$$
C_{N_{n}}=C_{N_{n}}^{f}+C_{N_{n}}^{v}
$$

An example of the force response during the pitching motion of an airfoil is shown in Figure 7, and the features mentioned above are noted. The tangential force coefficient $C_{T}$ needs to be estimated in order to find the lift and drag coefficients. The calculation of $C_{T}$ is based on Kirchhoff's approximation, and the dynamic separation point $f^{\prime \prime}$ is used:

$$
C_{T_{n}}=\eta C_{N_{\alpha}} \alpha_{n}^{2}\left(\sqrt{f_{n}^{\prime \prime}}-E_{0}\right)
$$


where $\eta$ and $E_{0}$ are empirical constants, $\eta=0.975$ and $E_{0}=0.15$ for the NACA0021 profile. When the normal and the tangential force coefficients are known, the lift $C_{L}$ and the drag $C_{D}$ coefficients are estimated as:

$$
\begin{aligned}
& C_{L_{n}}=C_{N_{n}} \cos \varphi_{n}+C_{T_{n}} \sin \varphi_{n} \\
& C_{D_{n}}=C_{N_{n}} \sin \varphi_{n}-C_{T_{n}} \cos \varphi_{n}+C_{D_{0}}
\end{aligned}
$$

where $C_{D_{0}}$ is the drag coefficient at the zero angle of attack and the relative wind flow angle $\varphi$ is obtained within the vortex model.

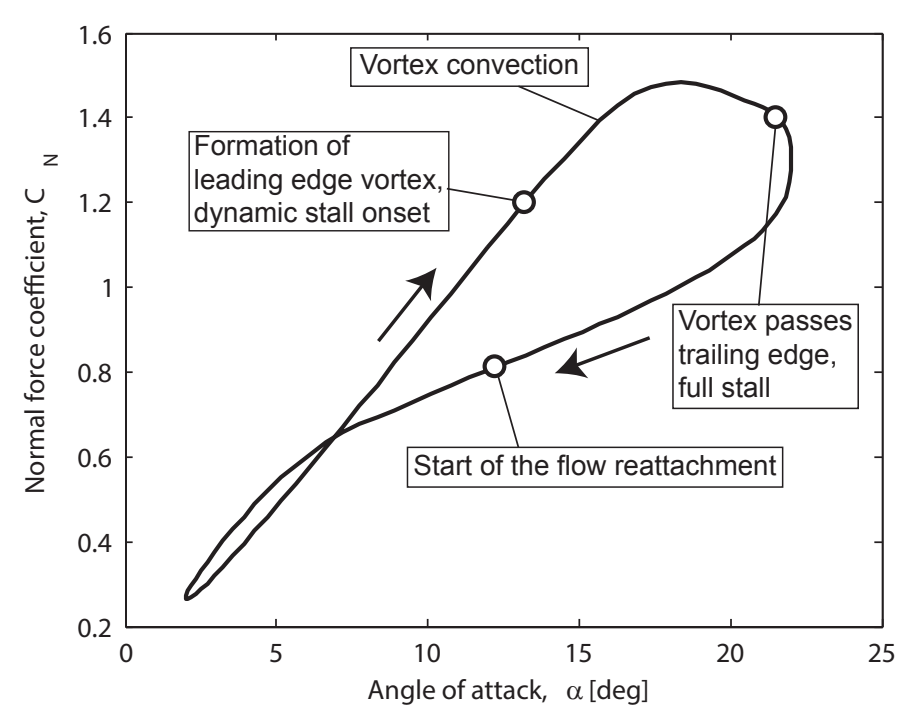

Figure 7. Normal force coefficient for the pitching NACA0021 airfoil, $\alpha=12+10 \sin (\omega t)$, $k=0.06, M=0.1, c=0.55 \mathrm{~m}$, where $k, M$ and $c$ are the reduced frequency, the Mach number and the chord length correspondingly.

\section{Modification Due to Vortex Shedding}

The presented dynamic stall model was tested in [25] for a pitching airfoil, where the flow direction was constant and the blade position was fixed. However, the blades perform circulatory motion during the operation of VAWTs, and thus, the model has to account for it. A schematic picture of the vortex shedding during the operation of a straight-bladed Darrieus turbine at low TSR is shown in Figure 8. Both the leading and trailing edge vortices are detached and swept away at Quadrant III. Consequently, the delay in the separation is not present in this region. To account for such flow conditions, the dynamic stall model is further modified as in [16]. The delay in the angle of attack and the vortex lift are set to zero to model fast vortex release:

$$
\text { quadrant III } \rightarrow \alpha^{\prime}=\alpha, C_{N}^{v}=0
$$




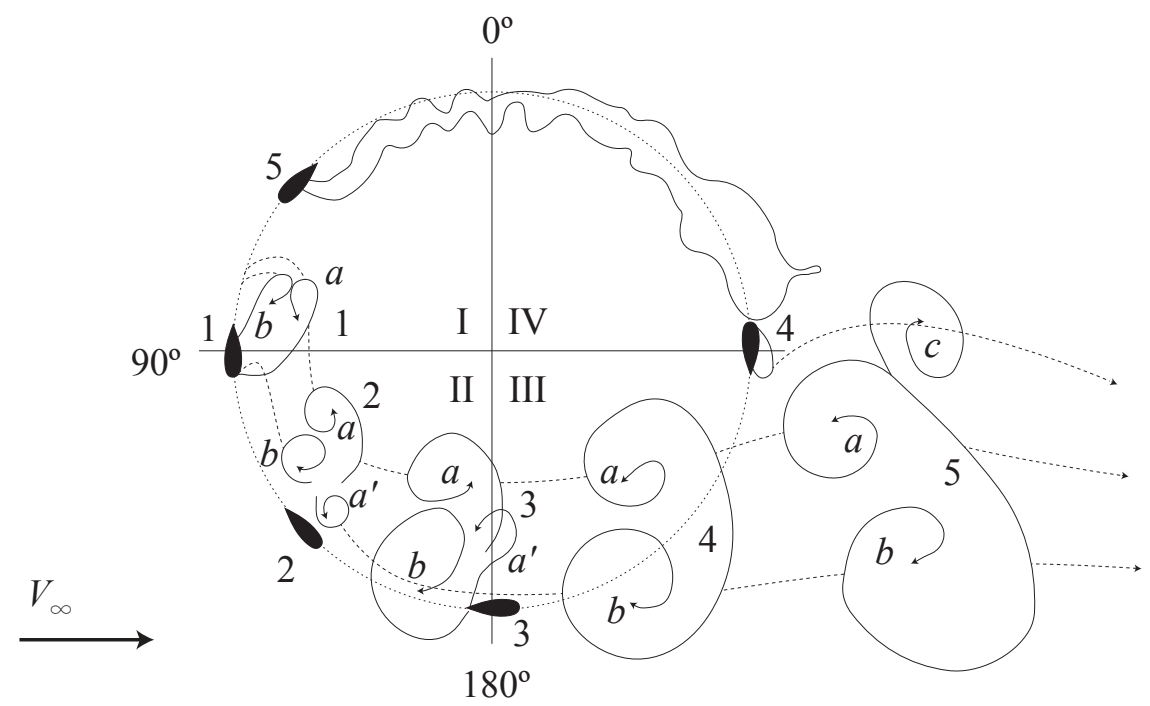

Figure 8. Dynamic vortex shedding for a straight-bladed vertical axis turbine operating in a towing tank at the tip speed ratio (TSR) of 2.14, obtained from [32]. $a, a^{\prime}, b$ and $c$ denote vortices.

\section{Results and Discussion}

This section presents the comparison of the simulation results against the measured data at different operational conditions. The discussions regarding the performance of the model are found at the end of this section.

The normal force response at low TSRs is presented in Figures $9-11$. The maximum magnitude of the $F_{N}$-response at the upwind is overestimated at $\lambda=1.84$ and $\lambda=2.26$, and the shape of the modeled $F_{N}$-curve at the downwind deviates from the measurements. The authors presume that the difference between the simulated and the measured values at these low TSRs is due to high magnitudes of the angle of attack. The accuracy of the dynamic stall model decreases with increased angle of attack, which is shown in $[25,29]$, where the dynamic stall model was tested against wind tunnel data for a single blade. As the angle of attack increases with decreased TSR, it is expected that the accuracy of the dynamic stall model should be limited at low TSRs. There is a positive offset of $F_{N}$ at $\theta=0^{\circ}$, which is mainly due to the blade pitch angle, which was chosen to even out the magnitude of $\alpha$ between the upwind and the downwind regions [18]. The value of the simulated $F_{N}$-offset is close to the measured one.

Figure 12 shows the $F_{N}$-response at $\lambda \approx 3$ for two different rotational speeds. The maximum magnitude of the $F_{N}$-curve is overestimated for $\Omega=65 \mathrm{rpm}$, similarly to the overestimation in Figures 9 and 10. The $F_{N}$-response at $\lambda \approx 3.45$ for $\Omega=50 \mathrm{rpm}$ and $\Omega=65 \mathrm{rpm}$ is presented in Figure 13. The results at these conditions are very similar to the results at $\lambda \approx 3$, although the model agrees better with experimental data at $\lambda \approx 3.45$. The measured $F_{N}$-response at $\lambda=3.44$ at $\Omega=65 \mathrm{rpm}$ has a drop in the downwind region at $225^{\circ}<\theta<325^{\circ}$, which is not predicted by the model. The discussions regarding the $F_{N}$-drop are found further in this section. 


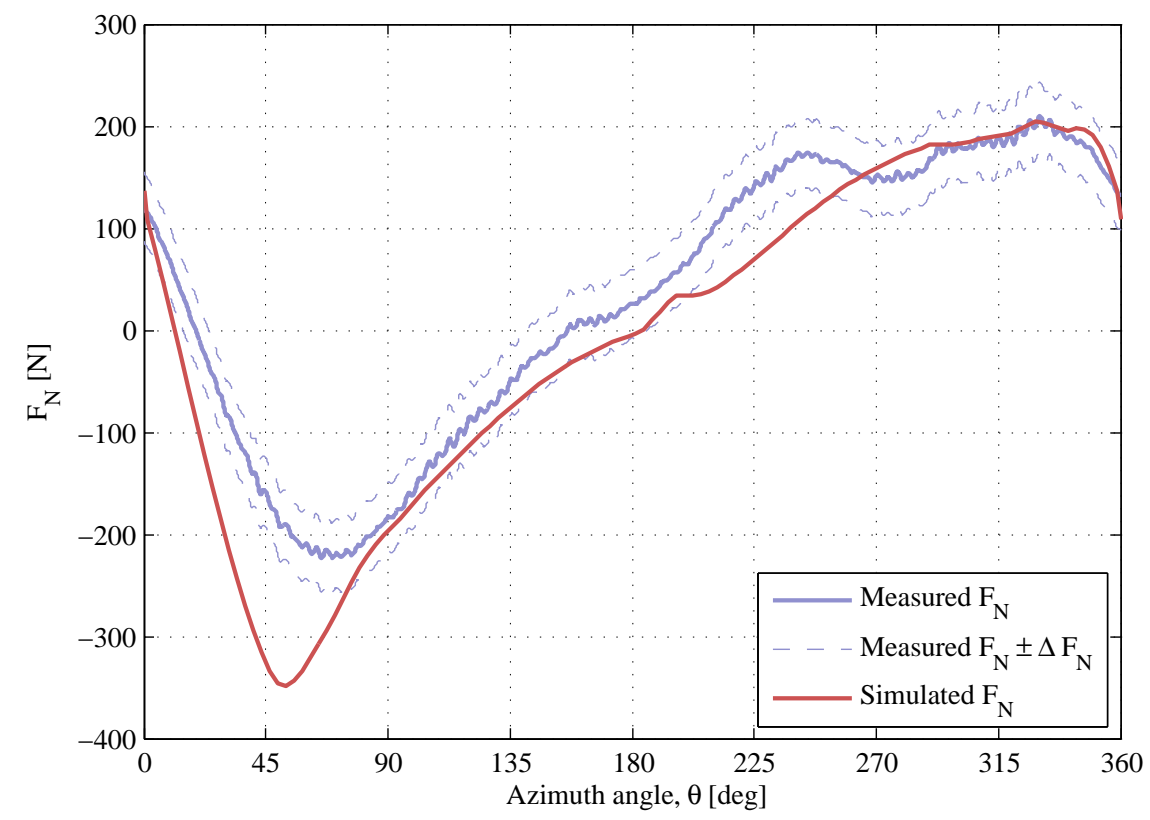

Figure 9. The normal force at $\lambda=1.84, \Omega=40.29 \mathrm{rpm}$. The air density and the kinematic viscosity are $\rho=1.25 \mathrm{~kg} / \mathrm{m}^{3}$ and $\nu=1.42 \cdot 10^{-5} \mathrm{~m}^{2} / \mathrm{s}$.

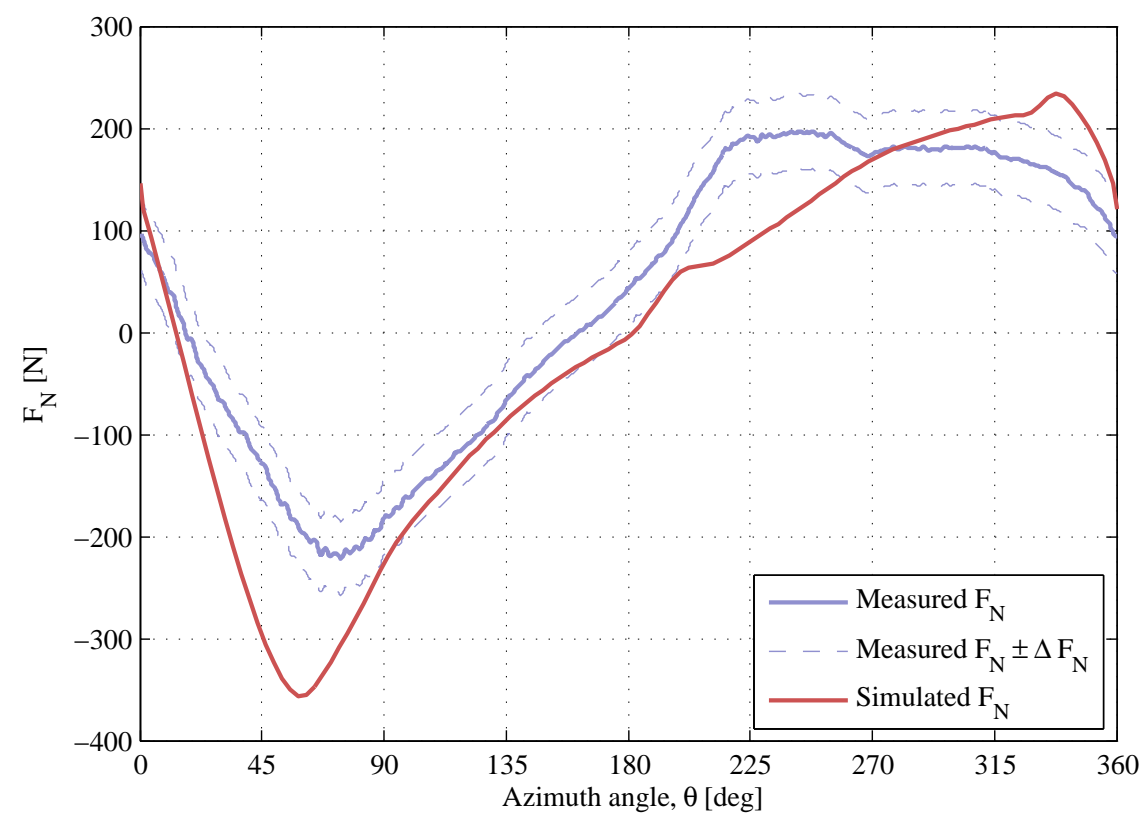

Figure 10. The normal force at $\lambda=2.26, \Omega=45.19 \mathrm{rpm}$. The air density and the kinematic viscosity are $\rho=1.25 \mathrm{~kg} / \mathrm{m}^{3}$ and $\nu=1.42 \cdot 10^{-5} \mathrm{~m}^{2} / \mathrm{s}$. 


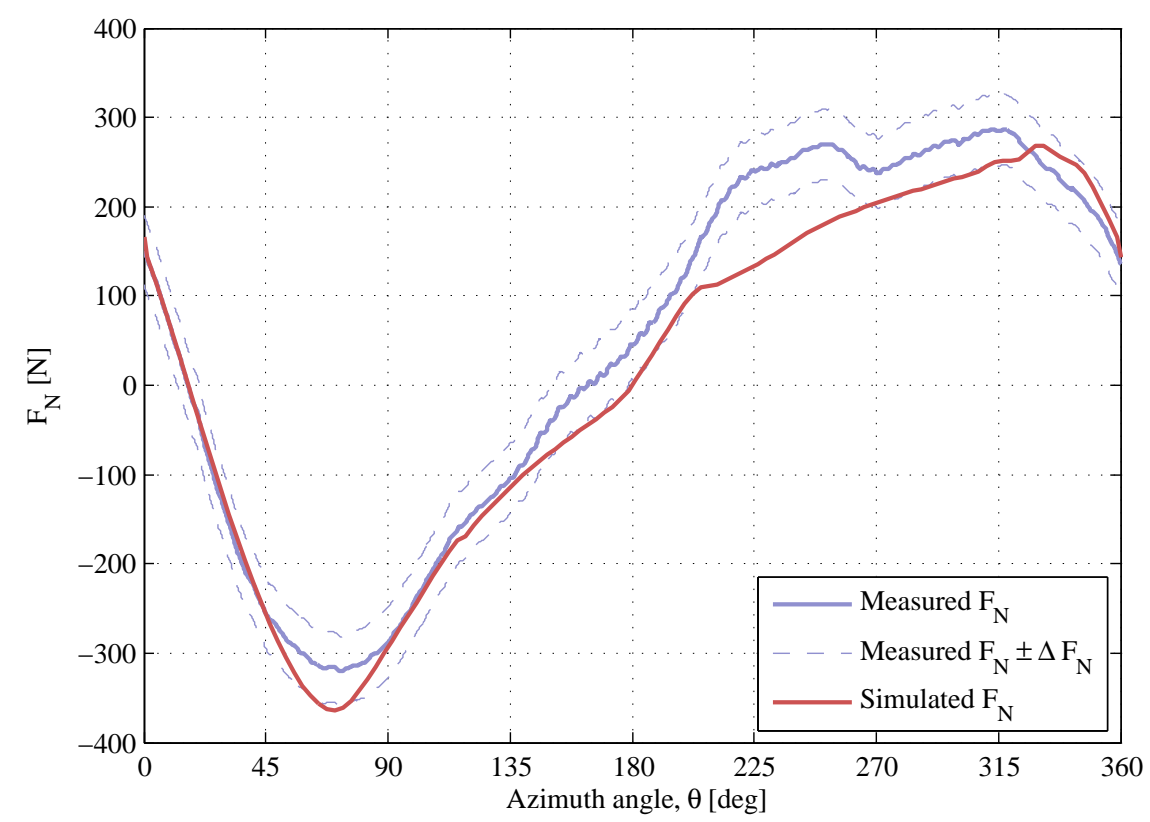

Figure 11. The normal force at $\lambda=2.85, \Omega=50.80 \mathrm{rpm}$. The air density and the kinematic viscosity are $\rho=1.27 \mathrm{~kg} / \mathrm{m}^{3}$ and $\nu=1.39 \cdot 10^{-5} \mathrm{~m}^{2} / \mathrm{s}$.

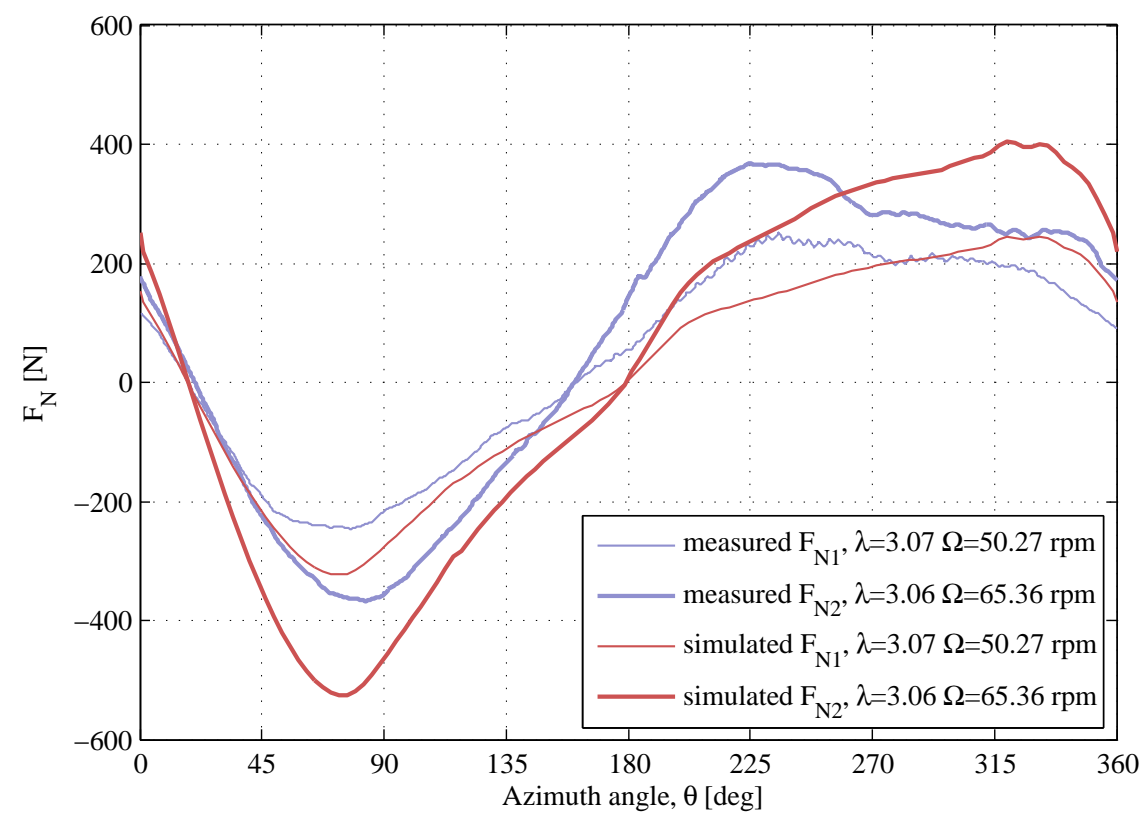

Figure 12. The normal forces at similar $\lambda$ and different $\Omega$. The air densities and the kinematic viscosities are $\rho_{1}=1.27 \mathrm{~kg} / \mathrm{m}^{3}, \rho_{2}=1.24 \mathrm{~kg} / \mathrm{m}^{3}$ and $\nu_{1}=1.39 \cdot 10^{-5} \mathrm{~m}^{2} / \mathrm{s}, \nu_{2}=1.45$. $10^{-5} \mathrm{~m}^{2} / \mathrm{s}$. 


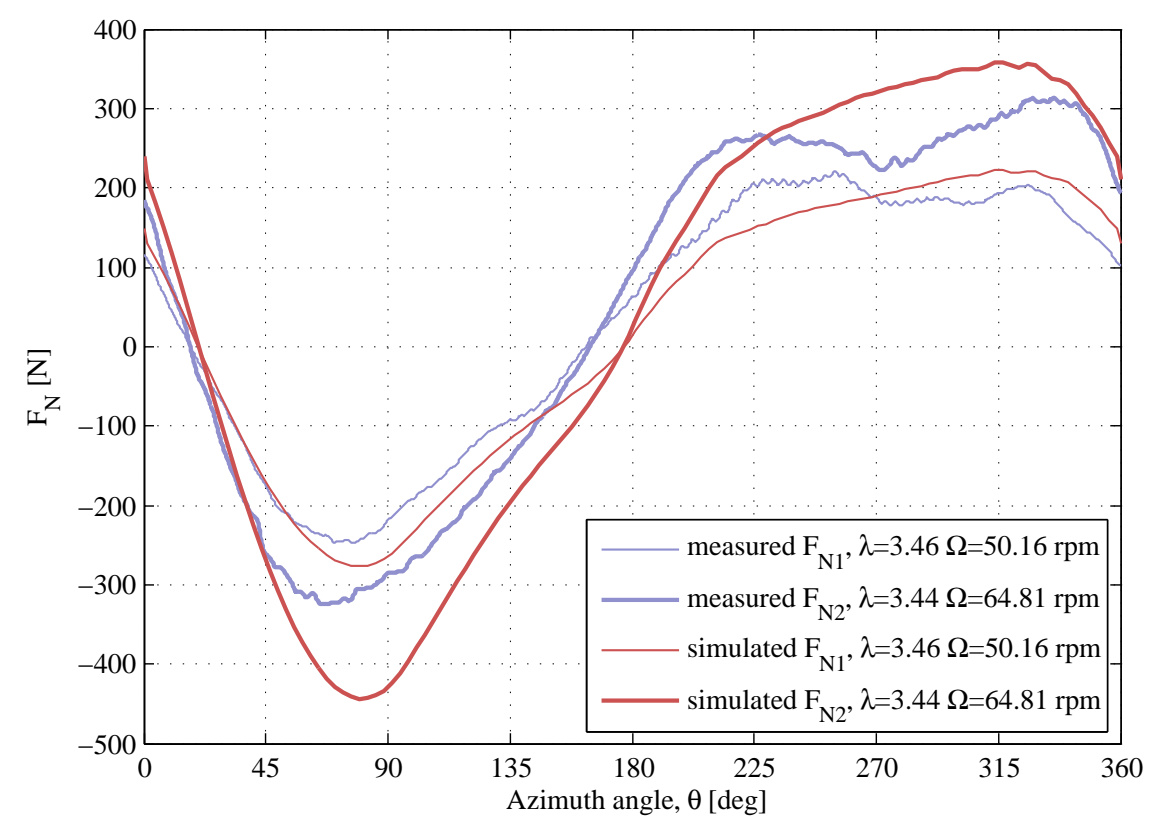

Figure 13. The normal forces at the similar $\lambda$ and different $\Omega$. The air densities and the kinematic viscosities are $\rho_{1}=1.28 \mathrm{~kg} / \mathrm{m}^{3}, \rho_{2}=1.24 \mathrm{~kg} / \mathrm{m}^{3}$ and $\nu_{1}=1.39 \cdot 10^{-5} \mathrm{~m}^{2} / \mathrm{s}$, $\nu_{2}=1.45 \cdot 10^{-5} \mathrm{~m}^{2} / \mathrm{s}$.

As the TSR increases, the maximum magnitude of the angle of attack decreases and the prediction of the blade forces becomes more accurate. The $F_{N}$-response at $\lambda=3.74$ is shown in Figure 14 . The simulated data are in a good agreement with the measured data except the $F_{N}$-drop at $235^{\circ}<\theta<330^{\circ}$, which is missed by the model. The $F_{N}$-response at $\lambda=3.88$ for two different rotational speeds is shown in Figure 15 . For both $F_{N}$-curves, the $F_{N}$-drop in the downwind is not predicted.

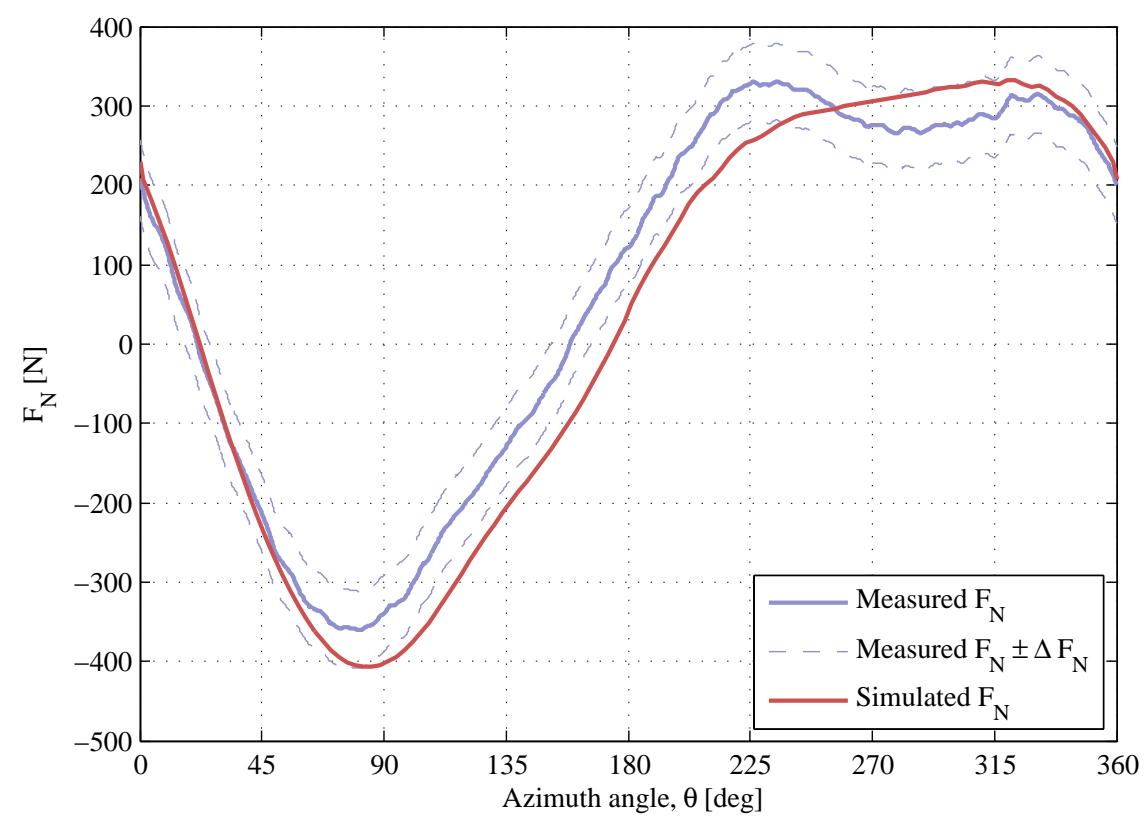

Figure 14. The normal force at $\lambda=3.74, \Omega=65.07 \mathrm{rpm}$. The air density and the kinematic viscosity are $\rho=1.24 \mathrm{~kg} / \mathrm{m}^{3}$ and $\nu=1.44 \cdot 10^{-5} \mathrm{~m}^{2} / \mathrm{s}$. 


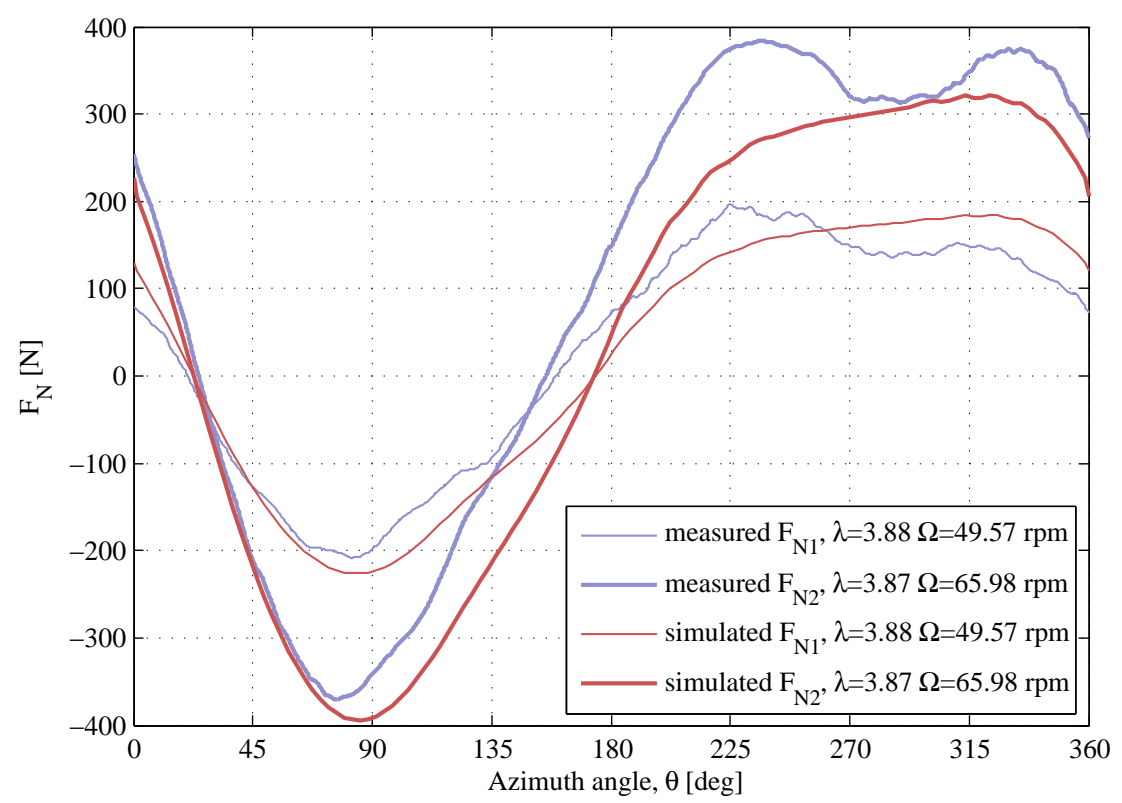

Figure 15. The normal forces at the similar $\lambda$ and different $\Omega$. The air densities and the kinematic viscosities are $\rho_{1}=1.28 \mathrm{~kg} / \mathrm{m}^{3}, \rho_{2}=1.24 \mathrm{~kg} / \mathrm{m}^{3}$ and $\nu_{1}=1.39 \cdot 10^{-5} \mathrm{~m}^{2} / \mathrm{s}$, $\nu_{2}=1.43 \cdot 10^{-5} \mathrm{~m}^{2} / \mathrm{s}$.

Two sets of the experimental data with almost identical operational conditions are compared against the simulated results in Figure 16. The shape of the measured $F_{N}$-curves is matching, but the magnitudes are slightly different. The maximum difference in the measured $F_{N}$ magnitudes is $\sim 50 \mathrm{~N}$, though the TSR is almost identical $\left(\lambda_{1}=3.94\right.$ and $\left.\lambda_{2}=4.00\right)$, and the difference in the air density is minor (see the notation to Figure 16). The model shows a close agreement, except that the $F_{N}$-drop at the downwind is not present.

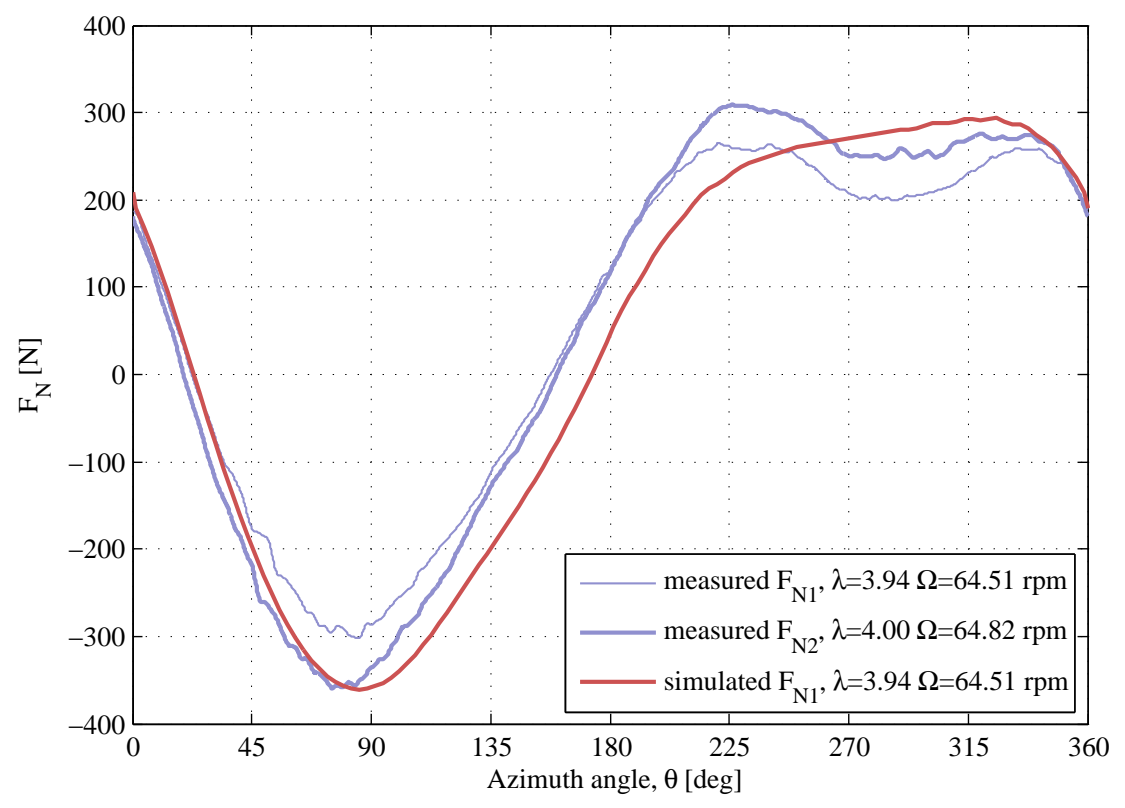

Figure 16. The normal forces for two different sets of data with almost identical $\lambda$ and $\Omega$. The air densities and the kinematic viscosities are $\rho_{1}=1.24 \mathrm{~kg} / \mathrm{m}^{3}, \rho_{2}=1.24 \mathrm{~kg} / \mathrm{m}^{3}$ and $\nu_{1}=1.44 \cdot 10^{-5} \mathrm{~m}^{2} / \mathrm{s}, \nu_{2}=1.45 \cdot 10^{-5} \mathrm{~m}^{2} / \mathrm{s}$. 
The results at the TSR of 4.6 are presented in Figure 17. At this high TSR, the flow expansion strongly affects the turbine aerodynamics. The model underestimates the maximum magnitude of the $F_{N}$-response in the downwind region. The aforementioned $F_{N}$-drop at the downwind is clearly observed at $\lambda=4.6$, and the model misses it.

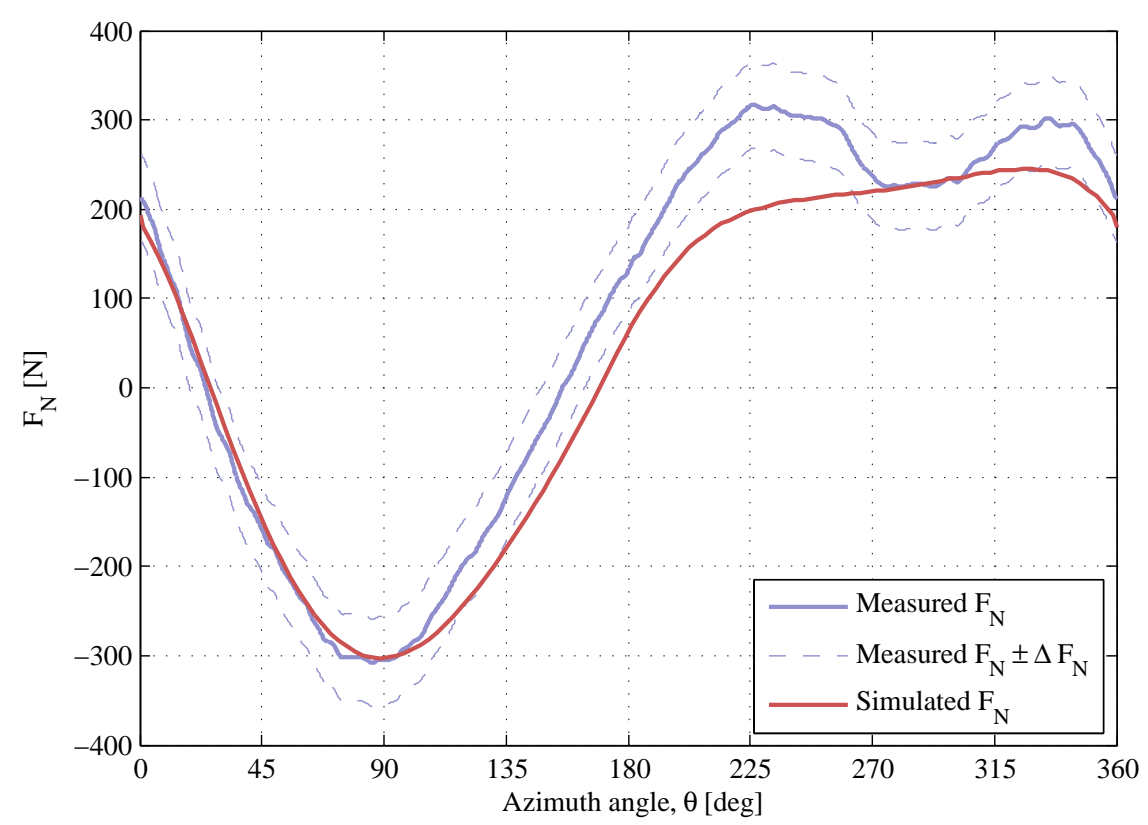

Figure 17. The normal force at $\lambda=4.57, \Omega=65.35 \mathrm{rpm}$. The air density and the kinematic viscosity are $\rho=1.25 \mathrm{~kg} / \mathrm{m}^{3}$ and $\nu=1.43 \cdot 10^{-5} \mathrm{~m}^{2} / \mathrm{s}$.

\section{General Discussion}

The presented simulations are in $2 \mathrm{D}$, while the measured data are in $3 \mathrm{D}$, and the contribution of the support arms is included in the measured forces. Therefore, it is expected that the presented model cannot reproduce the experimental results in great detail, especially where $3 \mathrm{D}$ effects are strong. The flow expansion in the simulation model is limited to the horizontal plane only, and the vertical expansion is omitted. This error should be most prominent at high TSRs, where the flow expansion is largest. Additionally, the current 2D model will not capture wind shear, which would cause a variation of the flow velocity, and hence, the TSR over the turbine height.

Over the whole range of the presented data, the model performs better in the upwind side. This is expected, since the dynamic stall vortex is not implemented in the flow field and the wake effects should be smaller in the upwind side. Furthermore, since the support arms are not included in the model, collision of the blade with vortices from the support arms cannot be reproduced. This is a possible contributing factor to the $F_{N}$-drop at $\lambda>3.4$, as the support arms can have a notable contribution to the wake. The $F_{N}$-drop is not expected to be due to the tower wake, since the tower diameter is considerably smaller than the region of the $F_{N}$-drop. This drop can also be caused by other three-dimensional effects, such as tip vortices, which are not included in the current model.

There are limitations of the dynamic stall model itself: it is assumed that the blade is a flat plate, and the flow velocity is constant during the change of the angle of attack [29]. Additionally, flow curvature is 
represented only through a correction in the angle of attack, while it can also influence the empirical constants of the dynamic stall model. These limitations should be considered when evaluating the performance of the model.

The maximum measurement error is estimated for every $F_{N}$-curve using Equation (5). Due to the high repeatability of the measured normal force, the shape of the $F_{N}$-curve is likely to remain, though the measurement error can change the scale of the $F_{N}$-response. This is observed when comparing two sets of data at almost identical operational conditions; Figure 16. Therefore, the measurement error has to be considered throughout the assessment of the simulation model.

The major advantage of the presented model is its computational speed: one simulation with 100 revolutions is in the order of minutes on a single core machine, which is much faster than simulations with 2D CFD models. A 3D vortex model does not have the previously-mentioned constraints with the flow expansion modeling and with the implementation of the support arms. However, the computational time of the existing 3D vortex models is still high, and the computational time of 3D CFD models can be a few months [1]. In this light, the presented simulation model can be used for the fast dimensioning of the turbine loads.

\section{Conclusions}

A two-dimensional vortex model for VAWTs was described. The simulation results on the normal forces were assessed against the new experimental data from the straight-bladed VAWT operated at an open site. The comparison is presented for a wide range of operational conditions. There is a drop in the normal force in the downwind region, which is more prominent at high TSR. The authors presume that this drop is due to three-dimensional effects, which are not implemented in the current model. The simulation model shows higher accuracy for the upwind region than for the downwind. At low TSRs, the model misses the measured results, which is expected to be due to the limitations of the dynamic stall model at the high magnitudes of the angle of attack. However, the simulated results agree well with the measured data at moderate and high TSRs, except the force drop in the downwind region. Although the model does not reproduce the experimental results in great detail, it shows a reasonable agreement with experimental data, and it can be used to simulate the maximum load limits on VAWTs at a low computational cost.

\section{Acknowledgments}

This project is conducted with the support of STandUP for Energy. The authors would like to acknowledge the J. Gust Richert foundation for the financial contribution to the equipment for the experiment. Morgan Rossander is acknowledged for experimental work and the estimation of measurement error.

\section{Author Contributions}

Eduard Dyachuk developed the dynamic stall model in its current implementation, obtained the results from experimental data and wrote the article. Anders Goude developed and described the vortex model and contributed to the data analysis. 


\section{Conflicts of Interest}

The authors declare no conflict of interest.

\section{References}

1. Li, C.; Zhu, S.; Xu, Y.L.; Xiao, Y. 2.5 D large eddy simulation of vertical axis wind turbine in consideration of high angle of attack flow. Renew. Energy 2013, 51, 317-330.

2. Paquette, J.; Barone, M. Innovative offshore vertical-axis wind turbine rotor project. Available online: http://wiki-cleantech.com/wind-energy/innovative-offshore-vertical-axis-wind-turbine-rotor -project (accessed on 1 October 2015).

3. Sutherland, H.J.; Berg, D.E.; Ashwill, T.D. A Retrospective of VAWT Technology; Technical Report SAND2012-0304; Sandia National Laboratories: Albuquerque, NM, USA, 2012.

4. Blusseau, P.; Patel, M.H. Gyroscopic effects on a large vertical axis wind turbine mounted on a floating structure. Renew. Energy 2012, 46, 31-42.

5. Goude, A. Fluid Mechanics of Vertical Axis Turbines. Simulations and Model Development. Ph.D. Thesis, Department of Engineering Sciences, Electricity, Uppsala University, Uppsala, Sweden, 14 December 2012.

6. Islam, M.; Ting, D.K.; Fartaj, A. Aerodynamic models for Darrieus-type straight-bladed vertical axis wind turbines. Renew. Sustain. Energy Rev. 2008, 12, 1087-1109.

7. Paraschivoiu, I. Wind Turbine Design-With Emphasis on Darrieus Concept; Presses Internationales Polytechnique: Montreal, QC, Canada, 2002.

8. Johnston, S.F. Proceedings of the Vertical Axis Wind Turbine (VAWT) Design Technology Seminar for Industry; Technical Report SAND80-0984; Sandia National Laboratories: Albuquerque, NM, USA, 1982.

9. Akins, R.E. Measurements of Surface Pressures on an Operating Vertical-Axis Wind Turbine; Technical Report SAND89-7051; Sandia National Laboratories: Albuquerque, NM, USA, 1989.

10. Oler, J.; Strickland, J.; Im, B.; Graham, G. Dynamic Stall Regulation of the Darrieus Turbine; Sandia National Laboratories: Albuquerque, NM, USA, 1983.

11. Strickland, J.H.; Webster, B.T.; Nguyen, T. A vortex model of the Darrieus turbine: An analytical and experimental study. J. Fluids Eng. 1979, 101, 500-505.

12. Ashuri, T.; van Bussel, G.; Mieras, S. Development and validation of a computational model for design analysis of a novel marine turbine. Wind Energy 2013, 16, 77-90.

13. Shires, A. Development and Evaluation of an Aerodynamic Model for a Novel Vertical Axis Wind Turbine Concept. Energies 2013, 6, 2501-2520.

14. Keinan, M. A Modified Streamtube Model for Vertical Axis Wind Turbines. Wind Eng. 2012, $36,145-180$.

15. Wang, K.; Hansen, M.O.L.; Moan, T. Model improvements for evaluating the effect of tower tilting on the aerodynamics of a vertical axis wind turbine. Wind Energy 2015, 18, 91-110.

16. Dyachuk, E.; Goude, A. Simulating Dynamic Stall Effects for Vertical Axis Wind Turbines Applying a Double Multiple Streamtube Model. Energies 2015, 8, 1353-1372. 
17. Rossander, M.; Dyachuk, E.; Apelfröjd, S.; Trolin, K.; Goude, A.; Bernhoff, H.; Eriksson, S. Evaluation of a blade force measurement system for a vertical axis wind turbine using load cells. Energies 2015, 8, 5973-5996.

18. Dyachuk, E.; Rossander, M.; Goude, A.; Bernhoff, H. Measurements of the Aerodynamic Normal Forces on a 12-kW Straight-Bladed Vertical Axis Wind Turbine. Energies 2015, 8, 8482-8496.

19. Kjellin, J.; Bülow, F.; Eriksson, S.; Deglaire, P.; Leijon, M.; Bernhoff, H. Power coefficient measurement on a $12 \mathrm{~kW}$ straight bladed vertical axis wind turbine. Renew. Energy 2011, 36, 3050-3053.

20. Co, C. Flow of Fluids Through Valves, Fittings, and Pipe; Number 410; Crane Company: Joliet, IL, USA, 1988.

21. Beale, J.T.; Majda, A. High order accurate vortex methods with explicit velocity kernels. J. Comput. Phys. 1985, 58, 188-208.

22. Greengard, L.; Rokhlin, V. A Fast Algorithm for Particle Simulations. J. Comput. Phys. 1987, 73, 325-348.

23. Goude, A.; Engblom, S. Adaptive fast multipole methods on the GPU. J. Supercomput. 2013, 63, 897-918.

24. Ramachandran, P.; Rajan, S.C.; Ramakrishna, M. A fast, two-dimensional panel method. SIAM J. Sci. Comput. 2003, 24, 1864-1878.

25. Dyachuk, E.; Goude, A.; Berhnoff, H. Simulating pitching blade with free vortex model coupled with dynamic stall model for conditions of straight bladed vertical axis turbines. J. Sol. Energy Eng. 2015, 137, 041008.

26. Goude, A.; Ågren, O. Simulations of a vertical axis turbine in a channel. Renew. Energy 2014, 63, 477-485.

27. Leishman, J.G.; Beddoes, T.S. A generalised model for airfoil unsteady behaviour and dynamic stall using the indicial method. In Proceedings of the 42nd Annual Forum of the American Helicopter Society, Washington, DC, USA, 2-5 June 1986; Westland Helicopters Ltd.: Yeovil, UK, 1986; pp. 243-265.

28. Leishman, J.G.; Beddoes, T.S. A semi-empirical model for dynamic stall. J. Am. Helicopter Soc. 1989, 34, 3-17.

29. Dyachuk, E.; Goude, A.; Bernhoff, H. Dynamic Stall Modeling for the Conditions of Vertical Axis Wind Turbines. AIAA J. 2014, 52, 72-81.

30. Sheng, W.; Galbraith, R.A.M.; Coton, F.N. A Modified Dynamic Stall Model for Low Mach Numbers. J. Sol. Energy Eng. 2008, 130, 1-10.

31. Sheldahl, R.E.; Klimas, P.C. Aerodynamic Characteristics of Seven Symmetrical Airfoil Sections through 180-Degree Angle of Attack for Use in Aerodynamic Analysis of Vertical Axis Wind Turbines; Technical Report SAND80-2114, Sandia National Laboratories: Albuquerque, NM, USA, 1981.

32. Brochier, G.; Fraunié, P.; Béguier, C.; Paraschivoiu, I. Water Channel Experiments of Dynamic Stall on Darrieus Wind Turbine Blades. AIAA J. Propul. Power 1986, 2, 445-449.

(C) 2015 by the authors; licensee MDPI, Basel, Switzerland. This article is an open access article distributed under the terms and conditions of the Creative Commons Attribution license (http://creativecommons.org/licenses/by/4.0/). 